\title{
Prognosis of Primary Osteosarcoma in Elderly Patients: A Comparison between Young and Elderly Patients
}

\author{
Hiroyuki Tsuchie $^{a}$ Makoto Emori $^{b}$ Hiroyuki Nagasawa ${ }^{a}$ Naohisa Miyakoshia \\ Yasutaka Murahashi $^{\mathrm{b}}$ Junya Shimizu ${ }^{\mathrm{b}}$ Emi Mizushima ${ }^{\mathrm{b}}$ Toshihiko Yamashitab \\ Yoichi Shimada ${ }^{a}$ \\ a Department of Orthopedic Surgery, Akita University Graduate School of Medicine, Akita, Japan; \\ ${ }^{\text {b}}$ Department of Orthopedic Surgery, Sapporo Medical University School of Medicine, Sapporo, Japan
}

\section{Significance of the Study}

- Although there have been some studies on the features of primary osteosarcoma in elderly patients, few have examined the long-term prognosis. We evaluated the clinical features of elderly patients with primary osteosarcoma and found that survival was relatively long in spite of a poor prognosis.

\section{Keywords}

Osteosarcoma · Elderly · Primary $\cdot$ Prognosis

\begin{abstract}
Objectives: Osteosarcoma is the most common malignant bone tumor in childhood. Although a poorer prognosis has been described in older patients, few reports have focused solely on primary osteosarcoma. We evaluated the clinical features of elderly patients with primary osteosarcoma. Materials and Methods: Ninety-four patients were included in this retrospective study, and we divided them into 2 groups (older patients and younger patients) based on a cut-off age of 40 years. The patients' information, including age, tumor type, location, presence of metastasis, American Joint Committee on Cancer (AJCC) stage, treatment-related factors, local and distant relapse, and outcome, was collected. We compared the clinical courses between the 2 groups in all
\end{abstract}

\section{KARGER}

E-Mail karger@karger.com www.karger.com/mpp

\section{(C) 2019 The Author(s)} Published by S. Karger AG, Basel

Karger

Open access

This is an Open Access article licensed under the Creative Commons Attribution-NonCommercial-4.0 International License (CC BY-NC) (http://www.karger.com/Services/OpenAccessLicense), applicable to the online version of the article only. Usage and distribution for commercial purposes requires written permission. and only deceased patients. Results: In all patients, the frequency of chemotherapy in the older group was significantly lower than in the younger group ( $p<0.001)$, and tumors were more frequent in axial bone in the older patients ( $p=$ $0.041)$. Only in patients with surgical treatment, histological effectiveness after chemotherapy in the older group was lower than in the younger group $(p=0.041)$. The older patients showed a poorer prognosis $(p=0.031)$. However, the 5 -year overall survival rate in the older patients was more favorable than that in the younger patients only among deceased patients $(p=0.032)$. Only the existence of metastasis affected the prognosis in older patients $(p=0.012)$. Conclusion: Primary osteosarcoma in elderly patients showed a high incidence of axial bone involvement, a low rate of chemotherapy, and resistance to chemotherapy. Although the final life prognosis is poor, survival may be relatively prolonged.

(c) 2019 The Author(s) Published by S. Karger AG, Basel 


\section{Introduction}

Osteosarcoma is the most common malignant bone tumor in childhood. However, this tumor has another peak of incidence in the elderly. Osteosarcoma patients older than 40 years comprised about $30 \%$ of all Japanese osteosarcoma patients from 2006 to 2015 [1]. Although the clinical and pathological features of osteosarcoma in adolescent patients have been well described, there are relatively few studies on the features of osteosarcoma in elderly patients [2-14]. Various reports have stated that older patients with osteosarcoma show a poorer prognosis than do adolescent patients, regardless of comprehensive treatment including surgery and chemotherapy. However, most of these studies included the sarcomatous transformation of Paget's disease of bone and secondary osteosarcoma after irradiation $[2-7,11]$. Paget's disease is uncommon in Asians, and elderly Asian patients with osteosarcoma are mostly considered to have primary osteosarcoma $[5,9]$. Although several reports on only primary osteosarcoma in elderly patients were published in recent years, few have examined the long-term prognosis. In addition, some reports included all patients and others included only patients who had surgery involving extremity bone and no metastasis, and the study objectives also differed [8-14].

The aim of this study was to analyze the clinical features and prognostic factors of elderly patients with osteosarcoma.

\section{Materials and Methods}

\section{Subjects}

We reviewed 112 cases of osteosarcoma involving the extremities or axial bones with or without metastasis in 2 hospitals between 1980 and 2016. Among them, we excluded patients with insufficient information (15 cases) or with secondary osteosarcoma due to Paget's disease or irradiated bones (3 cases); finally, a total of 94 consecutive patients were included in this retrospective study (48 males and 46 females, mean age: 31 years, range: $7-84$ ). Because many previous clinical trials of osteosarcoma in older patients set the inclusion criteria up to 40 years, we also divided patients into 2 groups at the age of 40 years [2, 3, 8-14]. In these sample, a total of 28 patients (31\%) were aged 40 years or older.

Patients' information was collected, including age, sex, tumor type, anatomical location of the tumor, metastasis at diagnosis, stage of the primary tumor, treatment-related factors, local and distant relapse, follow-up period, and outcome. The stage of the primary tumor was determined according to the staging system of the American Joint Committee on Cancer (AJCC), 7th edition [15]. We obtained some information about the type of local therapy, the rate of chemotherapy and its histological evaluation (Rosen and Huvos criteria), and the surgical margin (Enneking criteria) in those who had surgery as treatment-related factors [16]. In the absence of any events, the date of the last follow-up was considered an end-point. Overall survival (OS) was defined as the time period from the date of diagnosis to death or the last follow-up.

In our series, we compared these clinical courses and results between the patients aged 40 years or older (older group) and those aged 39 years or younger (younger group), and we assessed the differences between the 2 groups only in deceased patients. Furthermore, we compared only patients who underwent surgery with extremity bone osteosarcoma. In addition, we examined factors affecting the prognosis of older patients. This study was approved by the Institutional Review Board for Clinical Research at our university, and informed consent was obtained from all of the patients enrolled into this study.

\section{Statistical Analysis}

All values are expressed as the means $\pm \mathrm{SD}$. The Student $t$ test, the Welch $t$ test, and the $\chi^{2}$ test were used to compare the items between the 2 groups. The curve for OS was drawn according to the Kaplan-Meier method, and differences were analyzed by applying the log-rank test. Multivariate logistic regression analysis was used to identify prognostic factors. $p<0.05$ was considered statistically significant.

\section{Results}

The median follow-up period in all of the surviving patients was $138 \pm 88$ months (range: $12-372$ ), and that of the patients who died was $37 \pm 36$ months (range: 1-143). Histologically, 87 of the 94 patients had a conventional osteosarcoma (50 were osteoblastic, 14 were chondroblastic, and 11 were fibroblastic). Excluding the conventional type, 5 were telangiectatic osteosarcoma, 1 was small-cell osteosarcoma, and 1 was high-grade surface osteosarcoma. The sites of primary lesions were the extremities in 88 patients $(94.6 \%)$ and axial sites in $6(5.4 \%)$ as follows: distal femur in 38 , proximal tibia in 18 , proximal humerus in 8 , proximal femur in 8 , proximal fibula in 7 , pelvis in 5 , distal humeral in 2 , distal tibia in 2 , patella in 1 , proximal radius in 1 , distal radius in 1 , metacarpus in 1 , calcaneus in 1 , and sacrum in 1 patient. According to the AJCC staging system, 22 patients were classified as IIA, 57 were IIB, 3 were III, 9 were IVA, and 3 were IVB, and metastasis at diagnosis was observed in $12(12.8 \%)$ of all of the patients. Chemotherapy, such as neoadjuvant chemotherapy and chemotherapy after relapse, was administered to a total of 83 patients (88.3\%). The other 11 patients did not undergo chemotherapy due to patient refusal and/or internal comorbidity. Various chemotherapy regimens, including T-10, T-12, NSH-7, NECO95, JCOG0905, ifosfamide, ifosfamide-doxorubicin, methotrexate-ifosfamide, etoposide, and etoposide-ifosfamide, 
Table 1. Patient characteristics between the 2 groups in all patients and those with surgical treatment for extremity bone

\begin{tabular}{|c|c|c|c|c|c|c|}
\hline & \multicolumn{3}{|l|}{ All patients } & \multicolumn{3}{|c|}{ Patients with surgical treatment } \\
\hline & older group & younger group & $p$ value & older group & younger group & $p$ value \\
\hline Age, years & $62.5 \pm 13.8$ & $18.2 \pm 6.7$ & & $61.6 \pm 13.6$ & $17.8 \pm 6.7$ & \\
\hline Male/female ratio & $15 / 13$ & $33 / 33$ & 0.751 & $14 / 9$ & $31 / 30$ & 0.410 \\
\hline Extremity/axial ratio & $24 / 4$ & $64 / 2$ & 0.041 & $23 / 0$ & $61 / 0$ & - \\
\hline Conventional vs. others & & & 0.100 & & & 0.092 \\
\hline Conventional & $24(85.7)$ & $63(95.5)$ & & $20(87.0)$ & $59(96.7)$ & \\
\hline Telangiectatic & $2(7.1)$ & $3(4.5)$ & & $1(4.3)$ & $2(3.3)$ & \\
\hline Small cell & $1(3.6)$ & $0(0)$ & & $1(4.3)$ & $0(0)$ & \\
\hline High-grade surface & $1(3.6)$ & $0(0)$ & & $1(4.3)$ & $0(0)$ & \\
\hline Metastasis at diagnosis & & & 0.335 & & & 0.941 \\
\hline Present/none & $18 / 10$ & $65 / 1$ & & $16 / 7$ & $60 / 1$ & \\
\hline Surgery & & & 0.191 & & & - \\
\hline Present/none & $24 / 4$ & $62 / 4$ & & $23 / 0$ & $61 / 0$ & \\
\hline Surgical margin & & & 0.489 & & & 0.468 \\
\hline Adequate/inadequate & $23 / 1$ & $61 / 1$ & & $22 / 1$ & $60 / 1$ & \\
\hline Histological evaluation after chemotherapy & & & 0.083 & & & 0.041 \\
\hline Grades I-II/III-IV & $11 / 4$ & $29 / 31$ & & $11 / 3$ & $28 / 30$ & \\
\hline Unknown & 2 & 0 & & 2 & 0 & \\
\hline Metastasis after surgery & & & 0.246 & & & 0.289 \\
\hline Present/none & $13 / 11$ & $25 / 37$ & & $12 / 11$ & $24 / 37$ & \\
\hline Local recurrence & & & 0.242 & & & 0.132 \\
\hline
\end{tabular}

Values are expressed as numbers (\%) or means \pm SD unless otherwise stated.

were administered [17-21]. Surgery for the primary tumor was conducted in the 86 patients $(91.5 \%)$, and an adequate tumor-free margin was achieved in 84 patients (97.7\%). Information about histological evaluation after chemotherapy was available in 75 patients. According to Rosen and Huvos criteria, 29 patients were classified as grade I, 11 were grade II, 26 were grade III, and 9 were grade IV. Radiotherapy for the primary tumor was conducted for 8 patients (8.5\%), including heavy particle irradiation in 2 patients and proton beam radiotherapy in 2 patients. The outcomes of all of the patients were as follows: 47 patients had no evidence of disease and none of them were alive with disease, while 47 patients had died of disease (Table 2).

In all patients, the rate of chemotherapy in the older group $(64.3 \%)$ was lower than in the younger group $(98.5 \%)(p<0.001)$, and the tumor was common in axial bone in the older group $(14.3 \%)(p=0.041)$ (Table 1$)$. The outcome at the final follow-up in the older group revealed a poorer prognosis than in the younger group $(p=0.024)$ (Table 2). Kaplan-Meier OS curves between the 2 groups showed a significant difference $(p=0.031)$ (Fig. 1). Only in patients with surgical treatment for extremity bone, the rate of chemotherapy in the older group (69.6\%) was lower than in the younger group $(98.4 \%)(p<0.001)$, and histological effectiveness after chemotherapy in the older group was lower than in the younger group $(p=0.041)$ (Table 1). The outcome at the last follow-up in the older group showed a poorer prognosis than in the younger group ( $p=0.041$ ) (Table 2). Kaplan-Meier OS curves between the 2 groups showed no significant difference (Fig. 2). Only in deceased patients, the rate of chemotherapy in the older group (68.4\%) was lower than in the younger group $(100 \%)(p=0.003)$. The 5 - and 10 -year OS rates of the older group were 42.1 and $0 \%$, and those of the younger group were 14.3 and $3.6 \%$, respectively, 
Table 2. Prognostic comparison between the 2 groups

\begin{tabular}{|c|c|c|c|}
\hline & Older group & Younger group & $p$ value \\
\hline \multicolumn{4}{|l|}{ All patients } \\
\hline Outcome at the last follow-up & & & 0.024 \\
\hline Alive without disease & $9(32.1)$ & $38(57.6)$ & \\
\hline Alive with disease & $0(0)$ & 0 & \\
\hline Dead & $19(67.9)$ & $28(42.4)$ & \\
\hline 5 -year OS, \% & 62.3 & 62.9 & 0.972 \\
\hline 10 -year OS, \% & 31.3 & 53.2 & 0.129 \\
\hline \multicolumn{4}{|c|}{ Patients who had surgery involving extremity bone and no metastasis } \\
\hline Outcome at the last follow-up & & & 0.041 \\
\hline Alive without disease & $9(39.1)$ & $39(63.9)$ & \\
\hline Alive with disease & $0(0)$ & 0 & \\
\hline Dead & $14(60.9)$ & $22(36.1)$ & \\
\hline 5-year OS, \% & 68.4 & 65.5 & 0.816 \\
\hline 10 -year OS, \% & 38.5 & 56.8 & 0.244 \\
\hline
\end{tabular}

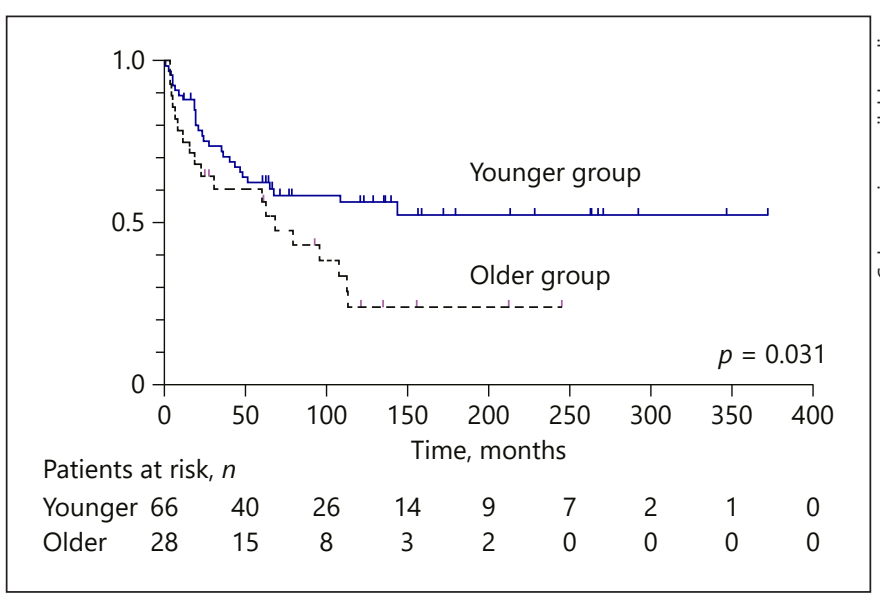

Fig. 1. Kaplan-Meier OS curves according to age in all patients. A significant difference was found between the 2 groups $(p=0.031)$.

showing a significant difference in 5 -year OS between the groups $(p=0.032)$ (Table 3$)$. Both in uni- and multivariate logistic regression analyses, only the presence of metastasis affected the prognosis in older patients $(p=0.012)$ (Table 4).

\section{Discussion}

Various outcomes regarding older patients with osteosarcoma have been reported with an unfavorable prognosis, and similar results were shown in our study. In addition, the rate of chemotherapy in the older pa-

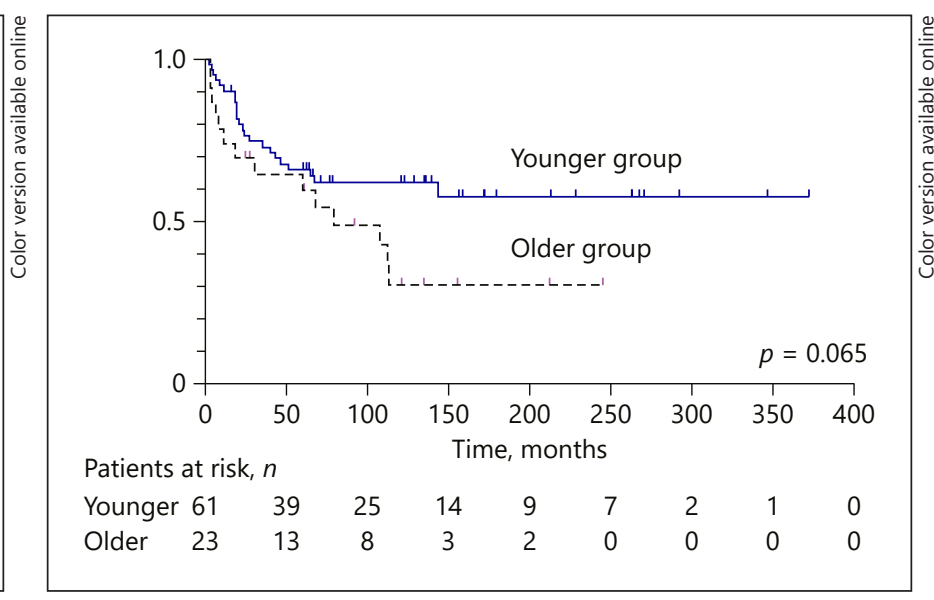

Fig. 2. Kaplan-Meier OS curves according to age in patients with surgical treatment. There was no significant difference between the 2 groups.

tients with osteosarcoma was lower than that in the younger patients, and osteosarcoma in the older patients was often identified in the axial bone. Several articles reported an increased incidence of axial bone involvement in older osteosarcoma patients [5-11]. A few reports excluding secondary osteosarcoma showed that the rate of occurrence in axial bone ranged from 20 to $49 \%$, although our study indicated a slightly low incidence (14.3\%) [8-10]. Jeon et al. [9] reported that the chemotherapy implementation rate was $72.7 \%$ in patients with osteosarcoma older than 40 years, and our study indicated a slightly low rate $(64.3 \%)$. In past studies excluding secondary osteosarcoma, the average age 
Table 3. Characteristics of deceased patients between the 2 groups

\begin{tabular}{|c|c|c|c|}
\hline & Older group, $\%$ & Younger group, $\%$ & $p$ value \\
\hline Patients & $19(40.4)$ & $28(59.6)$ & \\
\hline Age, years & $63.1 \pm 13.4$ & $19.1 \pm 6.8$ & \\
\hline Male/female ratio & $10 / 9$ & $14 / 14$ & 0.859 \\
\hline Extremity/axial ratio & $16 / 3$ & $26 / 2$ & 0.345 \\
\hline Metastasis at diagnosis & & & 0.919 \\
\hline Present/none & $5 / 14$ & $7 / 21$ & \\
\hline AJCC stage & & & 0.621 \\
\hline Stages I-II/III-IV & $13 / 6$ & $21 / 7$ & \\
\hline Chemotherapy & & & 0.003 \\
\hline Present/none & $13 / 6$ & $28 / 0$ & \\
\hline Surgery & & & 0.545 \\
\hline Present/none & $15 / 4$ & $24 / 4$ & \\
\hline Surgical margin & & & 0.423 \\
\hline Adequate/inadequate & $15 / 0$ & $23 / 1$ & \\
\hline Histological evaluation after chemotherapy & & & 0.320 \\
\hline Grades I-II/III-IV & $8 / 2$ & $15 / 9$ & \\
\hline Unknown & 1 & 0 & \\
\hline Local recurrence & & & 0.705 \\
\hline Present/none & $2 / 17$ & $4 / 24$ & \\
\hline 5 -year OS, \% & 42.1 & 14.3 & 0.032 \\
\hline 10 -year OS, \% & 0 & 3.6 & 0.405 \\
\hline
\end{tabular}

Values are expressed as numbers (\%) or means \pm SD unless otherwise stated.

Table 4. Univariate and multivariate logistic regression analysis of factors affecting the prognosis of older patients

\begin{tabular}{|c|c|c|c|c|c|c|}
\hline \multirow[t]{2}{*}{ Variables } & \multicolumn{3}{|c|}{ Univariate } & \multicolumn{3}{|c|}{ Multivariate } \\
\hline & OR & $95 \% \mathrm{CI}$ & $p$ & OR & $95 \% \mathrm{CI}$ & $p$ \\
\hline Age & 1.007 & $0.974-1.041$ & 0.675 & & & \\
\hline Sex & 1.002 & $0.403-2.489$ & 0.996 & & & \\
\hline \multicolumn{7}{|l|}{ Histological subtype } \\
\hline Conventional vs. others & 1.075 & $0.355-3.253$ & 0.898 & & & \\
\hline Location & 1.511 & $0.612-3.732$ & 0.371 & & & \\
\hline Metastasis at diagnosis & 4.546 & $1.448-14.270$ & 0.010 & 147.3 & $3.049-7,118.16$ & 0.012 \\
\hline AJCC stage & 1.589 & $1.050-2.404$ & 0.029 & 0.338 & $0.106-1.076$ & 0.066 \\
\hline Chemotherapy & 0.753 & $0.285-1.989$ & 0.566 & & & \\
\hline Surgery & 2.613 & $0.834-8.186$ & 0.099 & & & \\
\hline Histological evaluation after chemotherapy & 0.900 & $0.555-1.457$ & 0.667 & & & \\
\hline Metastasis after surgery & 4.482 & $1.448-13.873$ & 0.009 & 9.259 & $2.316-37.027$ & 0.002 \\
\hline Local recurrence & 0.380 & $0.087-1.668$ & 0.200 & & & \\
\hline
\end{tabular}

of the older patients in each study ranged from 53 to 61 years, and our study included patients with a high average (62.5 years) [8-10]. In our study, there were 12 patients $(43.1 \%)$ older than 70 years among the 28 patients with osteosarcoma older than 40 years, and the proportion of elderly patients was high. Chemotherapy for old- er patients is often contraindicated due to internal comorbidities. Therefore, this may be a reason why the rate of chemotherapy was low in this study.

Although some studies have examined the 5-year OS in elderly patients with primary osteosarcoma [8-14], few have investigated the long-term prognosis. Manoso 
et al. [12] reported the 10-year OS in elderly patients. However, their research was on data from surgically treated patients only. In past studies including only primary osteosarcoma, the 5-year OS in all patients ranged from 38.8 to $41.6 \%$, the 5 -year OS in patients with surgical treatment ranged from 44.3 to $71 \%$, and the surgically treated patients had a relatively high survival rate [8-14]. In our study, the 5-year OS in all patients was $62.3 \%$ and that in patients with surgical treatment was $68.4 \%$, and the surgically treated patients had a relatively high survival rate, as noted in the past reports. However, while the 10-year OS in all elderly patients dropped to $31.3 \%$, the 10 -year OS in elderly patients with surgical treatment was $48.5 \%$ and barely decreased. In addition, the 5-year OS only in deceased older patients was $42.1 \%$ and that in deceased younger patients was $14.3 \%$, and older patients showed a significantly higher survival rate. Based on these results, elderly patients often die within 10 years although they survive for 5 years. Some unknown factors affecting the survival period in elderly patients, such as a difference in the speed of metastatic lesion progression and sensitivity to radiotherapy, may be present. Further study is necessary to clarify this.

In previous reports, various factors affecting the prognosis of elderly osteosarcoma patients, such as the presence of metastasis at diagnosis, axial bone origin, and non-surgical treatment, have been described based on multivariate logistic regression analysis [8-10, 22]. Our study partially showed the same result, i.e., that the presence of metastasis markedly influences the prognosis. The site of occurrence may not have been one of the factors worsening the prognosis because of the small number of elderly patients with axial bone osteosarcoma in our study. Although they were not identified as prognostic factors in our study, a lower effectiveness of chemotherapy, nonspecific radiographic findings, and a high proportion of the fibroblastic type were reported as prognostic factors in elderly patients [2-14]. The role of chemotherapy in elderly patients with osteosarcoma remains controversial. However, in our study, the effect of chemotherapy was weak in the elderly patients, and we cannot deny the possibility that this low effectiveness of chemotherapy affected the prognosis.

This study had important strengths in showing novel findings of: (1) some clinical features and results of the long-term prognosis, and (2) a relatively long-term survival in deceased elderly patients with primary osteosarcoma. However, the limitations of this study should also be noted. First, one of the main limitations of this study was differences in treatment. Various chemotherapy regimens were administered to osteosarcoma patients, and radiotherapy was performed in some patients along with surgical treatment. However, the number of patients was small, making it difficult to strictly comply with the criteria for target patients. Second, we could not collect detailed information about comorbidity in this study. Elderly patients have more comorbidity, and this may have affected postoperative complications and the prognosis. We need to perform further detailed studies involving a larger number of elderly patients with primary osteosarcoma and collect comorbidity information. Finally, although we compared only deceased patients, elderly people generally have a poorer prognosis than young people, and a large bias in age may affect the results. However, it is important to examine the longterm prognosis of elderly patients in detail in countries with an aging population, such as Japan, and we would like to emphasize that this represents the first study to focus on the long-term prognosis of elderly patients. We were unable to conduct additional research examining the influence of this bias in this study, and so we need to consider this in a future study.

\section{Conclusion}

The present study showed some clinical features of primary osteosarcoma in elderly patients, such as a high incidence of axial bone involvement, a low rate of chemotherapy, and resistance to chemotherapy. Although the final life prognosis is poor, survival may be relatively long. We need to perform further studies to clarify the cause.

\footnotetext{
References

1 Japan Orthopaedic Association Muscloskeletal Tumor Committee/National Cancer Center. Bone and Soft Tissue Tumor Registry in Japan; 2015.

2 Naka T, Fukuda T, Shinohara N, Iwamoto Y, Sugioka Y, Tsuneyoshi M. Osteosarcoma versus malignant fibrous histiocytoma of bone in patients older than 40 years. A clinicopathologic and immunohistochemical analysis with special reference to malignant fibrous histiocytoma-like osteosarcoma. Cancer. 1995 Sep; 76(6):972-84.

3 Ek ET, Ojaimi J, Kitagawa Y, Choong PF. Outcome of patients with osteosarcoma over 40 years of age: is angiogenesis a marker of survival? Int Semin Surg Oncol. 2006 Mar; 3(1):7.
} 
4 Huvos AG. Osteogenic sarcoma of bones and soft tissues in older persons. A clinicopathologic analysis of 117 patients older than 60 years. Cancer. 1986 Apr;57(7):1442-9.

5 Okada K, Hasegawa T, Nishida J, Ogose A, Tajino T, Osanai T, et al. Osteosarcomas after the age of 50: a clinicopathologic study of 64 cases-an experience in northern Japan. Ann Surg Oncol. 2004 Nov;11(11):998-1004.

6 Longhi A, Errani C, Gonzales-Arabio D, Ferrari C, Mercuri M. Osteosarcoma in patients older than 65 years. J Clin Oncol. 2008 Nov; 26(33):5368-73.

7 Nishida Y, Isu K, Ueda T, Nishimoto Y, Tsuchiya $\mathrm{H}$, Wada $\mathrm{T}$, et al. Osteosarcoma in the elderly over 60 years: a multicenter study by the Japanese Musculoskeletal Oncology Group. J Surg Oncol. 2009 Jul;100(1):48-54.

8 Carsi B, Rock MG. Primary osteosarcoma in adults older than 40 years. Clin Orthop Relat Res. 2002 Apr;397:53-61.

9 Jeon DG, Lee SY, Cho WH, Song WS, Park $\mathrm{JH}$. Primary osteosarcoma in patients older than 40 years of age. J Korean Med Sci. 2006 Aug;21(4):715-8.

10 Iwata S, Ishii T, Kawai A, Hiruma T, Yonemoto $\mathrm{T}$, Kamoda $\mathrm{H}$, et al. Prognostic factors in elderly osteosarcoma patients: a multi-institutional retrospective study of 86 cases. Ann Surg Oncol. 2014 Jan;21(1):263-8.
11 Grimer RJ, Cannon SR, Taminiau AM, Bielack S, Kempf-Bielack B, Windhager R, et al. Osteosarcoma over the age of forty. Eur J Cancer. 2003 Jan;39(2):157-63.

12 Manoso MW, Healey JH, Boland PJ, Athanasian EA, Maki RG, Huvos AG, et al. De novo osteogenic sarcoma in patients older than forty: benefit of multimodality therapy. Clin Orthop Relat Res. 2005 Sep;438(438):110-5.

13 Bacci G, Ferrari S, Mercuri M, Longhi A, Fabbri N, Galletti S, et al. Neoadjuvant chemotherapy for osteosarcoma of the extremities in patients aged 41-60 years: outcome in 34 cases treated with adriamycin, cisplatinum and ifosfamide between 1984 and 1999. Acta Orthop. 2007 Jun;78(3):377-84.

14 Song WS, Kong CB, Jeon DG, Cho WH, Kim MS, Lee JA, et al. Prognosis of extremity osteosarcoma in patients aged 40-60 years: a cohort/case controlled study at a single institute. Eur J Surg Oncol. 2010 May;36(5):483-8.

15 Edge SB, Compton CC. AJCC Cancer Staging Handbook. 7th ed. New York: Springer; 2010.

16 Rosen G, Caparros B, Huvos AG, Kosloff C, Nirenberg A, Cacavio A, et al. Preoperative chemotherapy for osteogenic sarcoma: selection of postoperative adjuvant chemotherapy based on the response of the primary tumor to preoperative chemotherapy. Cancer. 1982 Mar;49(6):1221-30.

17 Rosen G, Marcove RC, Huvos AG, Caparros BI, Lane JM, Nirenberg A, et al. Primary osteogenic sarcoma: eight-year experience with adjuvant chemotherapy. J Cancer Res Clin Oncol. 1983;106(S1 Suppl):55-67.
18 Rosen G, Nirenberg A. Neoadjuvant chemotherapy for osteogenic sarcoma: a five year follow-up (T-10) and preliminary report of new studies (T-12). Prog Clin Biol Res. 1985; 201:39-51.

19 Wada T, Isu K, Takeda N, Usui M, Ishii S, Yamawaki S. A preliminary report of neoadjuvant chemotherapy NSH-7 study in osteosarcoma: preoperative salvage chemotherapy based on clinical tumor response and the use of granulocyte colony-stimulating factor. Oncology. 1996 May-Jun;53(3):221-7.

20 Iwamoto $\mathrm{Y}$, Tanaka $\mathrm{K}$, Isu $\mathrm{K}$, Kawai $\mathrm{A}$, Tatezaki S, Ishii T, et al: Multiinstitutional phase II study of neoadjuvant chemotherapy for osteosarcoma (NECO study) in Japan: NECO-93J and NECO-95J. J Orthop Sci. 2009;14:397-404

21 Iwamoto Y, Tanaka K. The activity of the Bone and Soft Tissue Tumor Study Group of the Japan Clinical Oncology Group. Jpn J Clin Oncol. 2012 Jun;42(6):467-70.

22 Colding-Rasmussen T, Thorn AP, Horstmann P, Rechnitzer C, Hjalgrim LL, KrarupHansen A, et al. Survival and prognostic factors at time of diagnosis in high-grade appendicular osteosarcoma: a 21 year single institution evaluation from east Denmark. Acta Oncol. 2018 Mar;57(3):420-5. 